\title{
Optimize the management of urological tube-related emergencies during the coronavirus disease 2019 (COVID-19) pandemic
}

\author{
Yang Luan ${ }^{1,2}$, Yan Zhang ${ }^{1,2}$, Kai Cui ${ }^{1,2}$, Fan Li $^{1,2}$, Baolong Qin ${ }^{1,2}$, Yajun Ruan ${ }^{1,2}$, Kun Tang ${ }^{1,2}$, \\ Hongyang Jiang ${ }^{1,2}$, Hao Li ${ }^{1,2}$, Xiaoyi Yuan ${ }^{1,2}$, Zhuo Liu ${ }^{1,2}$, Xiaming Liu ${ }^{1,2}$, Gan Yu ${ }^{1,2}$, Shengfei Xu ${ }^{1,2}$, \\ Ruibao Chen $^{1,2}$, Huan Yang ${ }^{1,2}$, Xiaolin Guo ${ }^{1,2}$, Xiaoyong Zeng ${ }^{1,2}$, Zhong Chen $^{1,2}$, Zhiqiang Chen ${ }^{1,2}$, \\ Zhiquan Hu ${ }^{1,2}$, Xiaodong Song ${ }^{1,2}$, Zhihua Wang ${ }^{1,2}$, Shaogang Wang ${ }^{1,2}$, Jihong Liu ${ }^{1,2}$, Tao Wang ${ }^{1,2}$ \\ ${ }^{1}$ Department of Urology, Tongji Hospital, Tongji Medical College, Huazhong University of Science and Technology, Wuhan, China; ${ }^{2}$ Institute of \\ Urology, Tongji Hospital, Tongji Medical College, Huazhong University of Science and Technology, Wuhan, China \\ Contributions: (I) Conception and design: Y Luan, Y Zhang, K Cui, F Li, B Qin, Y Ruan, K Tang, J Liu, T Wang; (II) Administrative support: X Guo, \\ X Zeng, Z Chen, Z Chen, Z Hu, X Song, Z Wang, S Wang, J Liu, T Wang; (III) Provision of study materials or patients: Y Luan, Y Zhang, K Cui, \\ F Li, B Qin, Y Ruan, K Tang, H Jiang, H Li, X Yuan, Z Liu, X Liu, G Yu, S Xu, R Chen, H Yang; (IV) Collection and assembly of data: Y Luan, Y \\ Zhang, K Cui, F Li, B Qin, Y Ruan, K Tang; (V) Data analysis and interpretation: Y Luan, Y Zhang, KaCui; (VI) Manuscript writing: All authors; (VII) \\ Final approval of manuscript: All authors. \\ Correspondence to: Tao Wang. Department of Urology, Institute of Urology, Tongji Hospital, Tongji Medical College, Huazhong University of Science \\ and Technology, 1095 Jiefang Avenue, Qiaokou District, Wuhan 430030, China. Email: twang@tjh.tjmu.edu.cn.
}

Background: To introduce and determine the value of optimized strategies for the management of urological tube-related emergencies with increased incidence, complexity and operational risk during the global spread of coronavirus disease 2019 (COVID-19).

Methods: All emergent urological patients at Tongji Hospital, Wuhan, during the period of January 23 (the beginning of lockdown in Wuhan) to March 23, 2020, and the corresponding period in 2019 were recruited to form this study's COVID-19 group and control group, respectively. Tongji Hospital has the most concentrated and strongest Chinese medical teams to treat the largest number of severe COVID-19 patients. Patients in the control group were routinely treated, while patients in the COVID-19 group were managed following the optimized principles and strategies. The case incidence for each type of tube-related emergency was recorded. Baseline characteristics and management outcomes (surgery time, secondary complex operation rate, readmission rate, COVID-19 infection rate) were analyzed and compared across the control and COVID-19 periods.

Results: The total emergent urological patients during the COVID-19 period was 42, whereas during the control period, it was 124 . The incidence of tube-related emergencies increased from $53 \%$ to $88 \%$ $(\mathrm{P}<0.001)$ during the COVID-19 period. In particular, the incidence of nephrostomy tube-related $(31 \%$ vs. $15 \%, \mathrm{P}=0.027)$ and single-J stent-related problems (19\% vs. $6 \%, \mathrm{P}=0.009)$ increased significantly. The mean surgery times across the two periods were comparable. The number of secondary complex operations increased from $12(18 \%)$ to $14(38 \%)(\mathrm{P}=0.028)$ during the COVID 19-period. The number of 2-week postoperative readmission decreased from $10(15 \%)$ to $1(3 \%)(\mathrm{P}=0.049)$. No participants contracted during the COVID-19 period.

Conclusions: Urological tube-related emergencies have been found to have a higher incidence and require more complicated and dangerous operations during the COVID-19 pandemic. However, the optimized management strategies introduced in this study are efficient, and safe for both urologists and patients.

Keywords: Coronavirus disease 2019 (COVID-19); urological tube-related emergency; surgery time; readmission; secondary complex operation

Submitted Aug 23, 2020. Accepted for publication Nov 24, 2020.

doi: $10.21037 /$ tau-20-1194

View this article at: http://dx.doi.org/10.21037/tau-20-1194 


\section{Introduction}

According to the latest data from Johns Hopkins University, more than 43 million individuals in 189 countries, including many medical professionals, have been diagnosed with coronavirus disease 2019 (COVID-19). More than 1.15 million patients had died by October 26, 2020. During the pandemic, medical resources are being fully used to ensure the prevention and control of COVID-19. Urology practices, including specialist clinics, outpatient procedures, and the use of operating theatres, have dramatically decreased $(1,2)$. It tends to result in ignorance and the delayed treatment of urological problems. Emergent urological problems need to be concerned and treated immediately; otherwise, they will cause serious renal failure or even worse. Common urinary emergencies include urinary retention, renal colic, hydronephrosis and anuria, urological tubes obstruction and dislodgment during the COVID-19 period. Most of these urological emergencies are directly related to or could be effectively solved by urological tubes.

However, routine management strategies may be not suitable at this time given the high exposure risk and operation complexity. Difficult urological tuberelated emergent operations may be increasing due to the inconvenience of seeing a doctor and complicated operations caused by treatment circumstances, protective measures, and the possibility of infection with COVID-19 during the pandemic. Therefore, it is important to pay closer attention to the management of tube-related urological emergencies. Common problems include: (I) difficulty inserting urinary catheter caused by severe prostatic hyperplasia, urethral stricture, and urinary tract damage; (II) failure to drainage urine due to bladder clots clogging; (III) difficulty dredging or replacing nephrostomy/cystostomy tube; (IV) difficulty dredging or replacing single-J stent; (V) double-J stent retentioninduced sickness. How to prepare a safe and effective plan to improve urological tube-related operations and how to balance COVID-19 prevention measures and emergency treatment during the pandemic merit investigation. To date, Tongji Hospital has the most concentrated and strongest medical teams from the whole country to treat the largest number of severe COVID-19 patients in Wuhan. Here, we investigate the case incidence changes in urological emergencies during the COVID-19 period. In addition, based on clinical practice, we optimize the management principles, strategies, and procedures for urological tube- related emergencies and evaluate their safety as well as effectiveness during the COVID-19 pandemic.

We present the following article in accordance with the TREND reporting checklist (available at http://dx.doi. org/10.21037/tau-20-1194).

\section{Methods}

\section{Patient population and data collection}

All emergent urological patients at Tongji Hospital, Tongji Medical College, Huazhong University of Science and Technology from January 23 (the beginning of lockdown in Wuhan) to March 23, 2020, and the corresponding period in 2019 were recruited to form this study's COVID-19 group and control group, respectively. Patients in the control group were routinely treated for urological tuberelated emergencies, while patients in the COVID-19 group were managed according to the optimized principles and strategies, as described in this article.

Data including baseline characteristics (gender, age, underlying diseases, tube retention time), emergent cases number and proportion, and management outcomes (surgery time, secondary complex operation rate, two-week postoperative readmission rate, COVID-19 infection rate) for urological tube-related emergencies were compared across groups to evaluate the value of the optimized strategies.

The study was conducted in accordance with the Helsinki Declaration (as revised in 2013) and approved by institutional research committee of Tongji Hospital (IRB Approval No. 215207-100). Written informed consent was obtained from all the patients.

\section{Optimized principles and strategies for the management of urological tube-related emergencies}

\section{General principles}

Evaluate the emergent situation in a non-contact way

Communicate with emergency doctors and patients to understand patients' medical histories and examination results using either voice or video call (e.g., WeChat). Evaluate the severity of each emergency by performing thorough examination. Develop preferred and alternative treatment plans before the preparation of the operating materials and entering the emergency department.

Screen for COVID-19 before performing any treatment

Conduct routine severe acute respiratory syndrome 


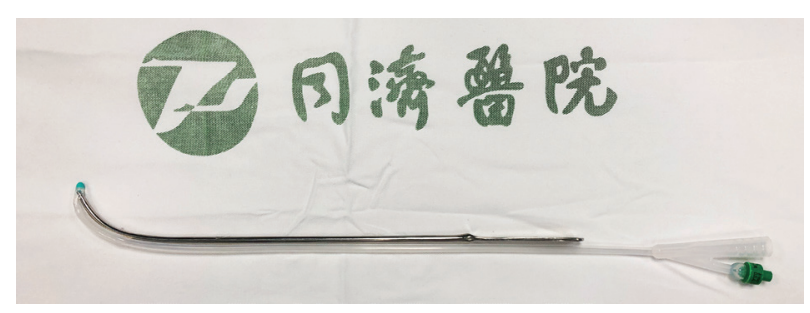

Figure 1 Arrangement of urethral probe and catheter in the integration method. Insert the front end of probe ( 8 or $10 \mathrm{Fr}$ ) into the side hole of catheter lubricated with sterile paraffin oil, and then insert them together into the urethra, which possess the smooth and tough features. Dilatation and insertion can be completed in one step.

coronavirus 2 (SARS-CoV-2) nucleic acid and antibody tests before performing any necessary urological inspections. Only life-threatening situations should be considered for immediate management.

\section{Determine the timing of treatment cautiously}

Regular replacement of urological tubes can be appropriately postponed until the pandemic is over as long as no obvious tube-related emergencies have occurred within the longest tube retention time. In case of tube obstruction, a patient can first try to flush the tube with sterile saline at home under the guidance of doctor by video call. For other tube-related problems, resolve current emergencies quickly and remove the etiologies when the pandemic is over.

\section{Select simple and low-exposure operations, prioritizing operations with high success rates and the avoidance of multiple operations}

Treatments should aim to rapidly relieve emergent conditions, provide urinary drainage, and protect renal function with minimally invasive methods. Treatments with simple steps, low accuracy requirements, short durations, and little body fluid contact are suggested to reduce potential exposure to the coronavirus. We suggest avoiding trying one method more than three times to prevent a prolonged operation. Operation methods and equipment with high success rates, rather than low cost, should be the first line of consideration. Carefully select procedures and check drainage patency postoperatively to reduce the need for multiple operations in the near future. Urological specialists with more than 5 years' work experience are best suited for performing these operations.

\section{Perform operations at emergency bedside when possible}

Perform operations in the emergency department to reduce transfers. Minimizing endoscopic and surgical procedures as far as the circumstances allow will help reduce the probability of cross-infection and excessive consumption of medical resources.

\section{Meet criteria for second-level protection}

Surgeons should wear second-level protective equipment before entering the emergency department and making contact with the patients. Minimize the number of surgeons and take only the necessary instruments into the operating area. Patients should wear medical protective masks if available.

The detailed management procedures for difficult urinary catheterization (Figures 1,2), bladder clot clogging (Figure 3), dislodgment or obstruction of nephrostomy/ cystostomy tube (Figure 4), dislodgment or obstruction of single-J stent (Figure 5), and sickness caused by double-J stent retention are listed in the Supplementary method section.

\section{Statistical analysis}

The case numbers (constituent ratio) across the two groups were statistically analyzed and compared using a two-tailed chi-square test. The age and time indices were presented as the mean \pm standard deviation (SD) and analyzed using the Student's $t$-test in Prism 6. A two-sided $\mathrm{P}$ value $<0.05$ was considered statistically significant.

\section{Results}

We gathered and analyzed all 2-month urological emergent clinic visit data from Tongji Hospital from January 23, 2020 (the beginning of lockdown in Wuhan) to March 23, 2020, and the corresponding period in 2019 (Table 1). The total number of urological emergent patients dropped to 42 , barely the one-third of the number in 2019. The incidence of tube-related emergencies reached $88 \%$ in 2020 , which is significantly higher than that of $53 \%$ in 2019 [relative risk (RR) 1.7, 95\% CI: 1.4-2.0, $\mathrm{P}<0.001]$. The percentage of non-tube-related emergencies, such as urinary tract injuries and testicular torsion, was dramatically decreased from $47 \%$ to $12 \%$.

Apart from the higher mean age seen during the COVID-19 period $(69 \pm 13$ vs. $55 \pm 8$ years old, $\mathrm{P}=0.042)$, the baseline characteristics of patients with tube-related emergencies across the two periods were unchanged. In terms of management outcomes, the mean surgery times $(17.7 \pm 4.2$ vs. $18.4 \pm 10.5 \mathrm{~min}, \mathrm{P}=0.950)$ were comparable. 


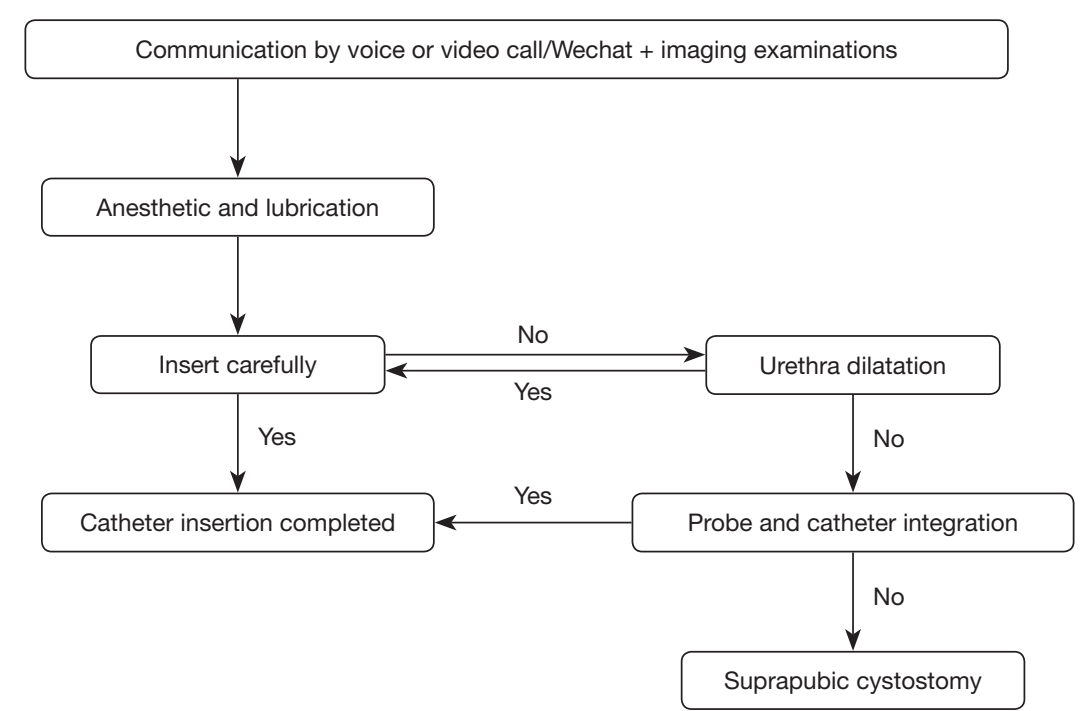

Figure 2 Medical procedures for difficulty inserting urinary catheterization. Fully understand patients' basic condition and medical history and judge the filling degree of bladder by ultrasonography. Anaesthetize and lubricate urethral mucosa before careful insertion of silica gel catheter with suitable size. If fail, urethra dilatation, probe and catheter integrative insertion, or suprapubic cystostomy can be chosen step by step.

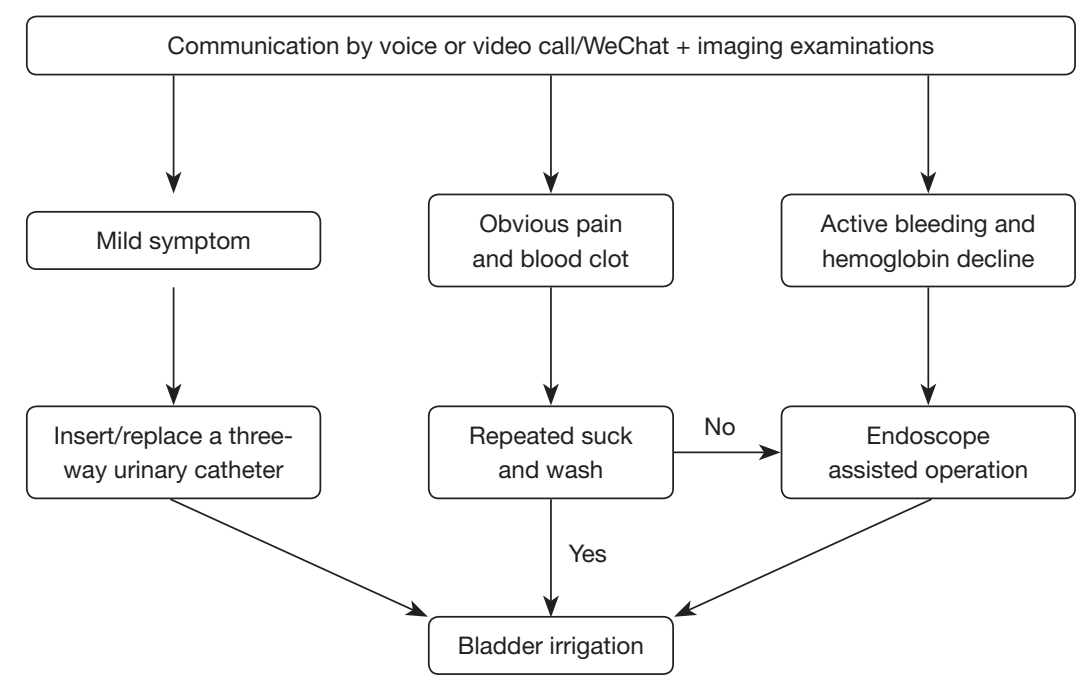

Figure 3 Medical procedures for bladder blood clots clogging. Judge the bleeding severity and source of blood clot by medical history, blood test and urinary imaging examination. Insert/replace a three-way urinary catheter and choose to suck and wash or endoscope assisted operation according to the severity of bleeding and obstruction.

The secondary complex operation rate, which reflects the difficulty of operation and includes all operations except direct tube/stent insertion or bladder irrigation, was higher in the COVID-19 group (38\%) than it was in control group (18\%) (RR 2.1, 95\% CI: 1.1-4.0, P=0.028). This mainly resulted from the increased complexity of replacing
nephrostomy/cystostomy/single-J tubes/stents during the COVID-19 period, although none of the specific tuberelated emergencies had statistical significance. However, the 2-week postoperative readmission rate was significantly lower in the COVID-19 group (3\%) than it was of control group (15\%) (RR 0.18, 95\% CI: 0.02-1.30, $\mathrm{P}=0.049$ ). Most 


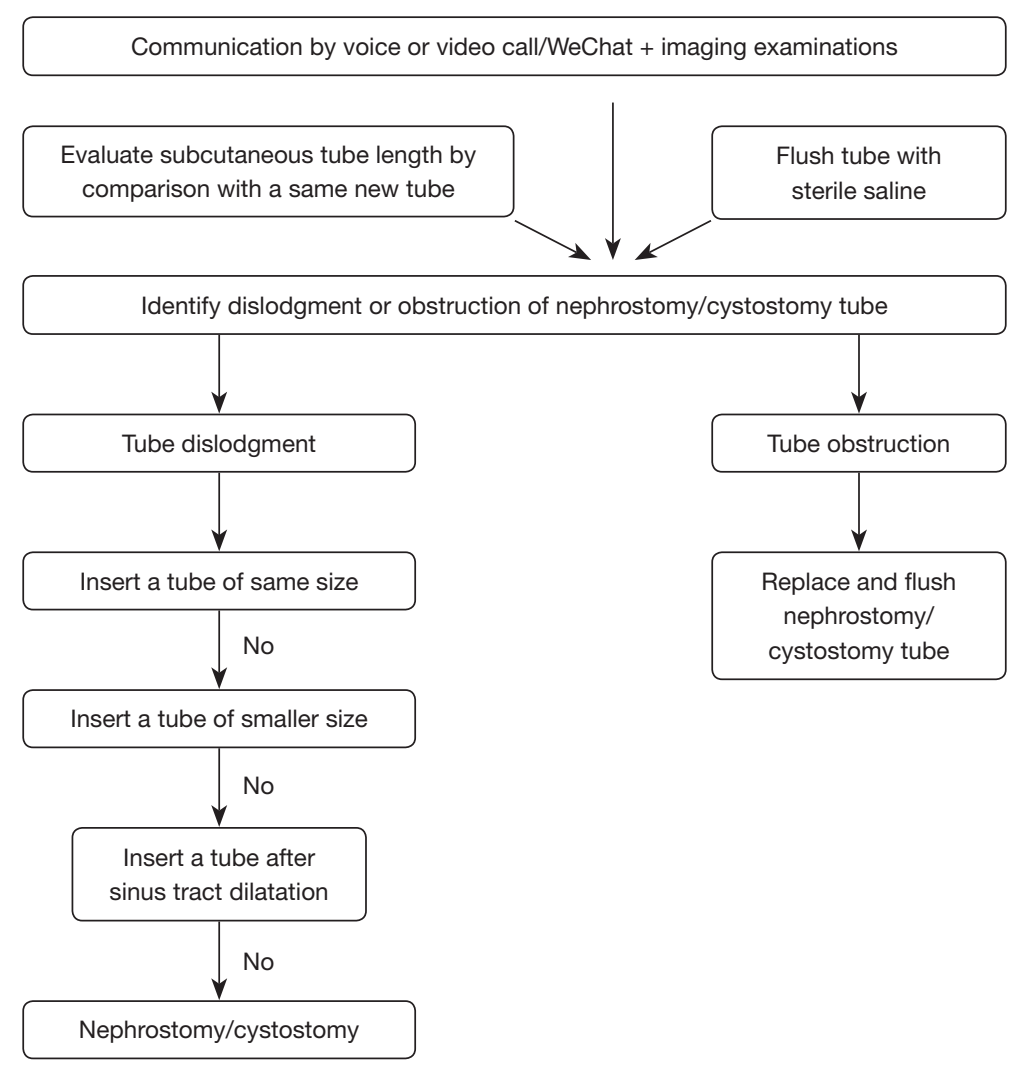

Figure 4 Medical procedures for dislodgment or obstruction of nephrostomy/cystostomy tube. Detect hydronephrosis, urine retention and the position of nephrostomy/cystostomy tube by medical history and imaging examination. Then, Identify the dislodgment or obstruction of nephrostomy/cystostomy tube. Insert a same or small size tube when nephrostomy/cystostomy tube is dislodged. If failed, try sinus tract dilatation with the guidance of a stiff guidewire or puncture again. Replace and flush nephrostomy/cystostomy tube when obstruction occur.

of readmissions that occurred in the control group were due to the recurrence of tube obstruction after a simple rinse of blocked tubes. Neither the surgeons nor the enrolled patients were found to be infected by SARS-CoV-2 within two weeks postoperatively.

Of all of the tube-related emergencies, nephrostomy tube-related ( $31 \%$ vs. $15 \%$, RR 2.0, 95\% CI: $1.1-3.7$, $\mathrm{P}=0.027)$ and single-J stent-related (19\% vs. $6 \%$, RR 3.4, 95\% CI: $1.3-8.7, \mathrm{P}=0.009)$ problems were the most significantly elevated in the COVID-19 group. In the COVID-19 group, the obstruction rates for nephrostomy (46\% vs. $11 \%, \mathrm{P}=0.022)$ and cystostomy tubes $(43 \%$ vs. $0 \%, \mathrm{P}=0.014)$ increased, while their dislodgment rates decreased. The incidence and complexity of difficult urinary catheterizations, bladder clots, and sickness caused by double-J stent retention were similar across both groups.

Of the 27 patients who experienced tubes obstruction (urinary catheter, nephrostomy tube, cystostomy tube, or
single-J stent), 13 patients (48\%) successfully dredged their tubes using sterile saline with video guidance from medical staff and did not need to visit the hospital. The remaining 14 patients $(52 \%)$ had especially severe or prolonged tube obstruction and were unable to dredge their tubes themselves. These patients ultimately went to emergency department for further management.

\section{Discussion}

Although COVID-19 seems to have a distant relationship with urinary diseases, most of urological patients are elderly individuals with relatively lower immunity and multiple underlying diseases. This makes them highly susceptible to SARS-CoV-2, and severe cases of it at that. In addition, COVID-19 is likely to cause renal failure once urinary obstruction occurs, and failure to promptly protect against or treat this condition could be life-threatening (3). 


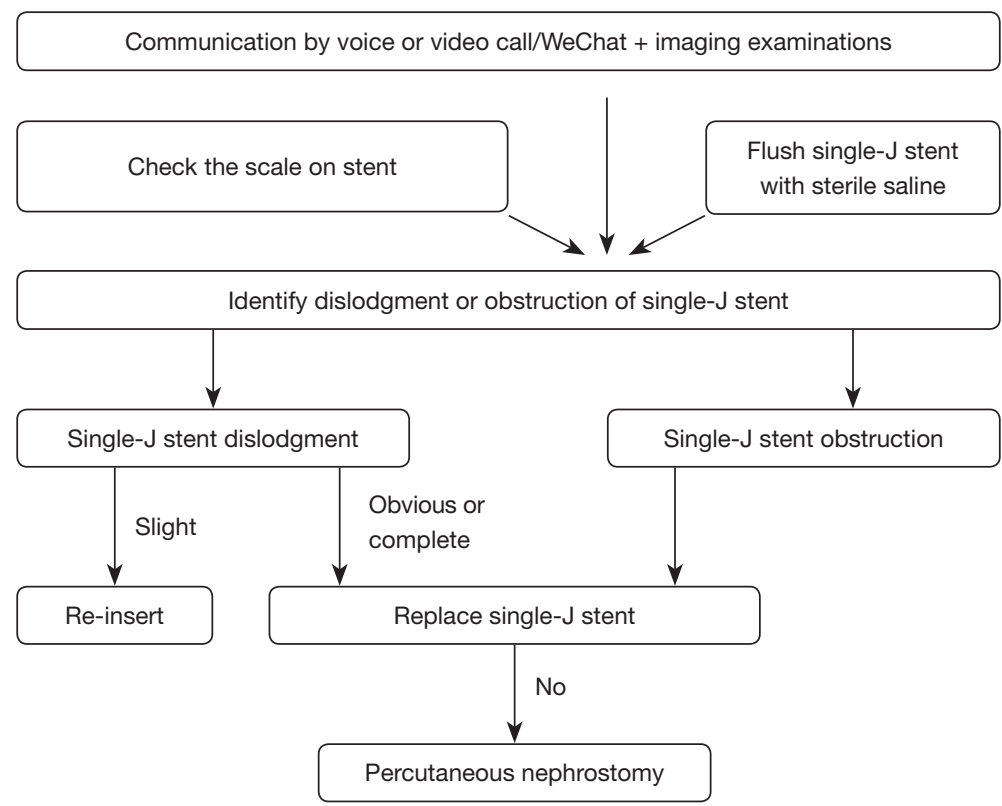

Figure 5 Medical procedures for dislodgment or obstruction of single-J stent. Detect hydronephrosis and the position of single-J stent by medical history and imaging examination. Then, identify dislodgment or obstruction of single-J stent. Slight dislodgment can be reinserted, while obvious/complete dislodgment or obstruction should replace a new single-J stent. If fail to insert a guidewire to pelvis, try to fix a bilateral open-ended ureteral catheter and detect the ureteral lumen with a hydrophilic guidewire. If all methods above do not work, percutaneous nephrostomy is suggested.

Our data showed an increased incidence of tube-related emergencies compared to other emergent problems during the COVID-19 period. In addition, the higher secondary complex operation rate suggests the increased complications associated with tube-related emergent operations during the pandemic, a finding which is in accordance with a recent report (4). These situations may be attributed to the delayed replacement of tubes, less social activity, the postponement of treatment due to a fear of infection as well as the inconvenience of traveling to the hospital, and interference with operational accuracy caused by protective equipment. Therefore, these patients merit attention.

The optimized strategies give priority to operation success rates and the avoidance of multiple operations. Many optimized procedures, such as the direct replacement of obstructed tubes instead of simple rinses, the thoroughly flushing out of clots or sediment, and the checking of patency after operation, ensured operational effectiveness and lower readmission rates within a short period of time postoperatively. This in turn reduce the exposure risk for both doctors and patients. It is important to note that when doctors followed the optimized operation principles and strategies, the mean surgery time during the COVID-19 period was comparable to that of the control period, although the doctors' vision and movement were greatly impacted by the protective equipment and the operational complexity was elevated. The optimized strategies compensated for surgery time by promoting adequate evaluation and preparation, the selection of simple and reliable operations, and reduced attempts to use methods with low success rates. These data demonstrated that the optimized strategies can guarantee the success rate and efficiency of urological tube-related emergent operations.

SARS-CoV-2 is known to be transmitted through the respiratory tract, close contact, and aerosolized particles $(5,6)$. Recently it has been isolated from the urine of COVID-19 patients, suggesting that contact with patients' urine may also become a potential route of infection (7). Some studies have shown that $30-60 \%$ of the SARS$\mathrm{CoV}$-2-infected population may be asymptomatic and tested negatively during the early phase of infection; however, these individuals may still be capable of infecting others (7-11). Operational urologists are exposed to a high infection risk by coming into contact with patients' breath/blood/urine while performing urological tuberelated procedures. Therefore, the optimized strategies 
Table 1 Contemporary comparison of urological emergencies in Tongji Hospital during COVID-19 and control period

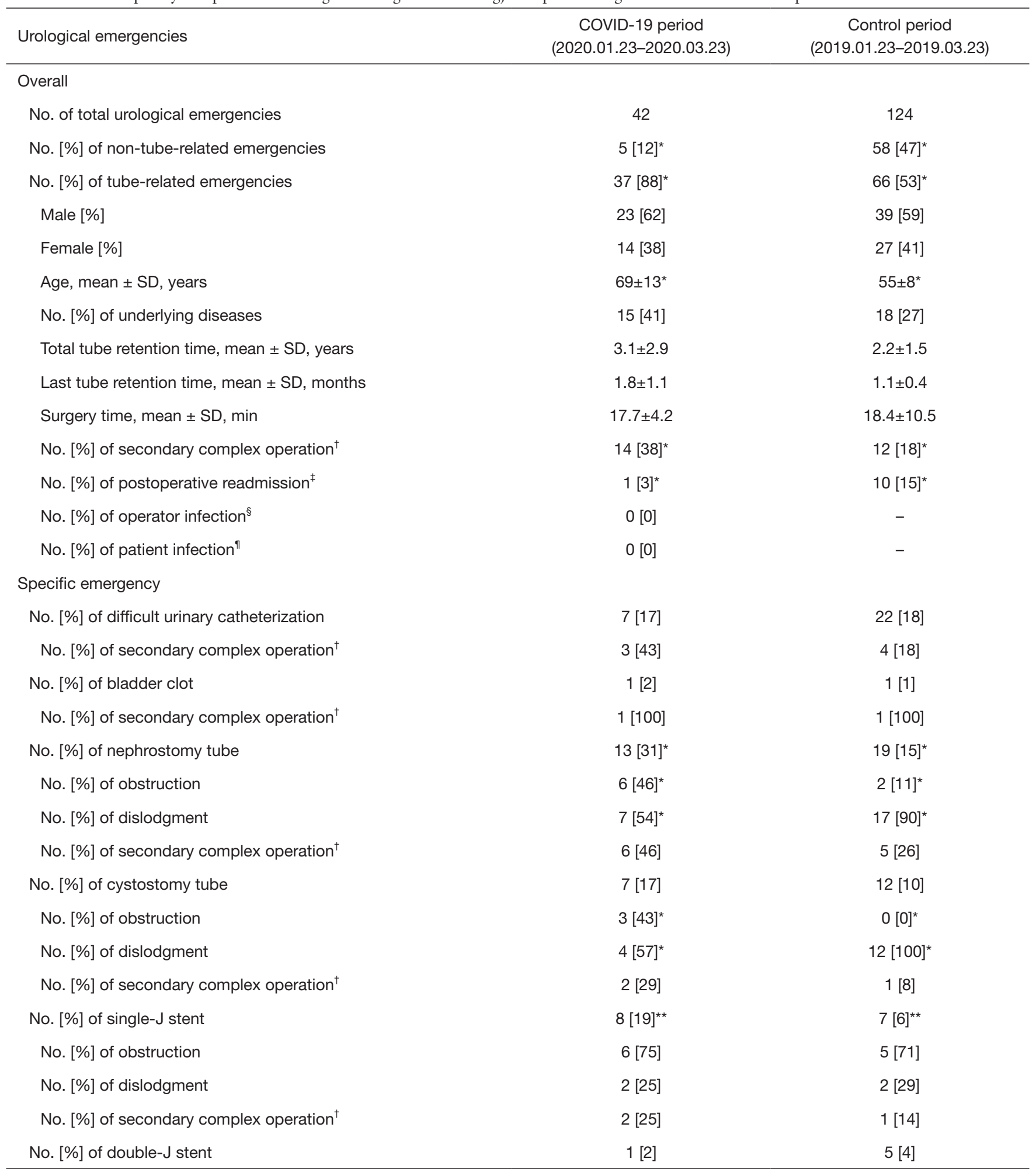

${ }^{*}, \mathrm{P}<0.05 ;{ }^{* \star}, \mathrm{P}<0.01$ of comparison between the COVID-19 and control period. ${ }^{\dagger}$, all operations except direct tube/stent insertion or bladder irrigation (including dilatation, wire or endoscope guidance, puncture, clot suck, bladder hemostasis, etc.); ${ }^{\ddagger}$, readmission to emergency department within two weeks after operations; ${ }^{\S}$, suspected or confirmed COVID-19 infection of operator within two weeks after operations; ", suspected or confirmed COVID-19 infection of patient within two weeks after operations. 
suggest standard second-level protection when entering the contaminated areas and making contact with patients during the pandemic. Performing simple and low-exposure operations, controlling surgery time, and decreasing the need for multiple operations also help to prevent surgeons and patients from becoming infected with SARS-CoV-2. The lack of COVID-19 infections among all participants in this study indicate the safety of the optimized strategies.

Some limitations existed in this study. First, in order to decrease the exposure risk of all of our participants, we did not set control group with patients under routine management during COVID-19 period. This prevented us from making additional comparisons. Second, the study focused on a single hospital and used a relatively small sample size and short follow-up period. Third, the cost effectiveness property of the optimized strategies was not evaluated.

\section{Conclusions}

Urological tube-related emergencies have been found to have a higher incidence and require more complicated and dangerous for operations during the COVID-19 period. However, the principles, strategies, and procedures of the optimized management introduced in this study are efficient, and safe for both urologists and patients during the COVID-19 pandemic.

\section{Acknowledgments}

We thank the Emergency Department of Tongji hospital for its coordination in patients management.

Funding: None.

\section{Footnote}

Reporting Checklist: The authors have completed the TREND reporting checklist. Available at http://dx.doi. org/10.21037/tau-20-1194

Data Sharing Statement: Available at http://dx.doi. org/10.21037/tau-20-1194

Conflicts of Interest: All authors have completed the ICMJE uniform disclosure form (available at http://dx.doi. org/10.21037/tau-20-1194). The authors have no conflicts of interest to declare.
Ethical Statement: The authors are accountable for all aspects of the work in ensuring that questions related to the accuracy or integrity of any part of the work are appropriately investigated and resolved. The study was conducted in accordance with the Helsinki Declaration (as revised in 2013) and approved by institutional research committee of Tongji Hospital (IRB Approval No. 215207100). Written informed consent was obtained from all the patients.

Open Access Statement: This is an Open Access article distributed in accordance with the Creative Commons Attribution-NonCommercial-NoDerivs 4.0 International License (CC BY-NC-ND 4.0), which permits the noncommercial replication and distribution of the article with the strict proviso that no changes or edits are made and the original work is properly cited (including links to both the formal publication through the relevant DOI and the license). See: https://creativecommons.org/licenses/by-nc-nd/4.0/.

\section{References}

1. Chan MC, Yeo SEK, Chong YL, et al. Stepping Forward: Urologists' Efforts During the COVID-19 Outbreak in Singapore. Eur Urol 2020;78:e38-9.

2. Ahmed K, Hayat S, Dasgupta P. Global challenges to urology practice during the COVID-19 pandemic. BJU Int 2020;125:E5-6.

3. Wang Y, Yang C, Xiao J, et al. Suggestions on the diagnosis and control of urology in the context of pneumonitis with new coronavirus infection. Journal of Modern Urology 2020;2:95-100.

4. Puliatti S, Eissa A, Eissa R, et al. COVID-19 and urology: a comprehensive review of the literature. BJU Int 2020;125:E7-14.

5. Ong SWX, Tan YK, Chia PY, et al. Air, Surface Environmental, and Personal Protective Equipment Contamination by Severe Acute Respiratory Syndrome Coronavirus 2 (SARS-CoV-2) From a Symptomatic Patient. JAMA 2020;323:1610-2.

6. WHO Infection Prevention and Control Guidance for COVID-19. Available online: https://www.who.int/ emergencies/diseases/novel-coronavirus-2019/technicalguidance/infection-prevention-and-control

7. General Office of the National Health and Health Commission. Notice on Printing and Distributing the Pneumonia Diagnosis and Treatment Plan for the New 
Coronavirus Infection (Trial Version 7) [EB/OL]. Available online: http://www.nhc.gov.cn

8. Wang C, Liu L, Hao X, et al. Evolving Epidemiology and Impact of Non-pharmaceutical Interventions on the Outbreak of Coronavirus Disease 2019 in Wuhan, China. medRxiv 2020. doi: 10.1101/2020.03.03.20030593.

9. Nishiura H, Kobayashi T, Suzuki A, et al. Estimation of the asymptomatic ratio of novel coronavirus infections

Cite this article as: Luan Y, Zhang Y, Cui K, Li F, Qin B, Ruan Y, Tang K, Jiang H, Li H, Yuan X, Liu Z, Liu X, Yu G, Xu S, Chen R, Yang H, Guo X, Zeng X, Chen Z, Chen Z, Hu Z, Song X, Wang Z, Wang S, Liu J, Wang T. Optimize the management of urological tube-related emergencies during the coronavirus disease 2019 (COVID-19) pandemic. Transl Androl Urol 2021;10(1):466-474. doi: 10.21037/tau-20-1194
(COVID-19). Int J Infect Dis 2020;94:154-5.

10. Wölfel R, Corman VM, Guggemos W, et al. Virological assessment of hospitalized patients with COVID-2019. Nature 2020;581:465-9.

11. He X, Lau EHY, Wu P, et al. Temporal dynamics in viral shedding and transmissibility of COVID-19. Nat Med 2020;26:672-5. 


\section{Management procedure of each tube-related emergency}

\section{Difficult urinary catheterization}

Fully understand patients' basic condition, medical history (such as severe prostatic hyperplasia, urethral stricture, and trauma-induced urinary tract damage), and judge the filling degree of bladder by ultrasonography in emergency department to assist diagnosis and operation choice. Before the operation, inject $2 \%$ lidocaine into urethra to fully infiltrate and anaesthetize the urethral mucosa. Cover and fully lubricate the catheter sterile paraffin oil to make it smooth. Select an appropriate type of catheter according to the diameter of patient's external urethral orifice (usually ranging from $1 / 3$ to $1 / 2$ the diameter of the external urethral orifice) and the reason of urine retention. In general, the catheter made of silica gel (14-16 Fr) and emulsion (16$18 \mathrm{Fr}$ ) are commonly chosen for most patients, and the silica gel catheter is more suitable for those patients with severe prostatic hyperplasia or urethral stricture because the tough material can partly resist enlarged adenoma or narrow urethra. In case of failed insertion by common method, urethral dilatation by probe covered with sterile paraffin oil can be tried gently from small to large size gradually. After successfully retaining the last probe in the urethra for three minutes, repeat to try insertion. Generally, $18-\mathrm{Fr}$ probe is enough for 14-16 Fr catheter, and 20-Fr probe for 16-18 Fr catheter. If still fail to insert the catheter yet, try probe and catheter integrative insertion (Figure 1). If all methods above do not work, suprapubic cystostomy is recommended (Figure 2) (12-14).

\section{Bladder clot clogging}

Distinguish and confirm the cause of bladder clots clogging by medical history (such as history of hematuria, urological surgery, genitourinary cancer, and coagulation disorders) before operation. Routine blood test and urinary ultrasonography or CT scan can be utilized in emergency department to help judge the bleeding severity (such as active bleeding or the size of blood clot) and source of blood clot. For patients with mild bladder clots clogging, immediately insert or replace a three-way urinary catheter, and conduct continuous bladder irrigation with sterile saline. Adjust the speed of irrigation according to the color of outflow liquid to keep it clear. For patients with obvious pain, phloroglucinol, indomethacin, and some another similar drugs can be used to relieve analgesic and spasmolytic effects. If an obvious blood clot blocks the urinary catheter, use a syringe to suck and wash quickly and repeatedly followed by continuous bladder irrigation. If the blood clot in the bladder is too big to suck it out, or combined with possible active bleeding judged by the color of outflow liquid or routine blood test, we suggest endoscope-assisted clots clearance and careful hemostasis, followed by continuous bladder irrigation (Figure 3).

\section{Dislodgment or obstruction of nephrostomy/cystostomy tube}

Initially, fully understand patients' basic condition and medical history (such as diseases treated by nephrostomy/ cystostomy, duration of nephrostomy/cystostomy, the time since poor drainage or dislodgment, symptoms and so on). Ask the emergency department clinicians for ultrasonography/X-ray/computed tomography (CT) scan to detect hydronephrosis, urine retention and the position of the nephrostomy/cystostomy tube. Then, identify the dislodgment or obstruction of nephrostomy/cystostomy tube. If imaging examinations show the tube out of renal collecting system/bladder or awareness of shortening of subcutaneous tube length by comparison with a new tube, it could be partial or complete dislodgement. If the tube locates in the right position of renal collecting system/ bladder and difficult to flush with sterile saline, it could be obstruction by sediments. Note to exclude prerenal or renal anuria. If the nephrostomy/cystostomy tube is dislodged, try to directly insert a new tube of the same or smaller size (12 Fr minimum) (15). For nephrostomy tube insertion, the abdomen-elevated prone position is taken to keep the sinus tract straight, as is the standard position for percutaneous nephrostomy. For cystostomy tube insertion, patients take the supine position. The sinus tract orientation is estimated based on puncture site and imaging examinations. If direct insertion does not work, try to insert a tube after the sinus tract dilatation with the guidance of a stiff guidewire. Pull out the tube and insert a 6-Fr ureteral catheter carefully according to the estimated sinus tract orientation. Generally, urine will flow out when the ureteral catheter enters into renal collecting system/bladder. Remove the tip of ureteral catheter and the open-ended catheter is placed into the collecting system/bladder to guide the insertion of a stiff guidewire. Then, remove the ureteral catheter followed by sinus tract dilatation and tube insertion with the guidance of the stiff guidewire. If fail to insert a ureteral catheter into renal collecting system/bladder, percutaneous nephrostomy/cystostomy to insert a new tube is suggested 
to perform with the guidance of ultrasonography $(16,17)$. If the nephrostomy/cystostomy tube is obstructed, a new tube should be replaced immediately. Use sterile saline to irrigate the tube repeatedly to wash out crystals or pyuria in collecting system/bladder (Figure 4). Antibiotics are appropriately used based on the severity of urinary infection.

\section{Dislodgment or obstruction of single- $\mathcal{7}$ stent}

Initially, fully understand patients' basic condition and medical history (such as diseases treated by cutaneous ureterostomy, duration of cutaneous ureterostomy, the time since poor drainage or dislodgment, symptoms and so on). Ask the emergency department clinicians for ultrasonography/X-ray/CT to detect hydronephrosis and the position of the single-J stent. If imaging examinations show single-J stent out of renal collecting system or awareness of shortening of intraureteral stent length based on scales on the single-J stent, dislodgment of single-J stent can be identified. If the single-J stent is in the correct position but difficult to flush with sterile saline, it could be obstructed by sediments (18). Slight dislodgment can be solved by immediate re-insertion, while obvious or complete dislodgment can be solved by inserting a new single-J stent. A flexible-ended hydrophilic guidewire is recommended for the procedure. The guidewire slides into ureter till sense of slight resistance. Then pull out the original single-J stent. Cut off the tip of a new single-J stent and insert it with the guidance of the hydrophilic guidewire. If guidewire is obstructed in the middle of ureter and cannot reach renal pelvis, it may be attributed to stone formation around stents, false passages and stenosis by repeated replacements, local excessive curvature of ureter, and external compression (such as intestines, tumors). Try to insert and fix a bilateral open-ended ureteral catheter at the obstructed site and insert a hydrophilic guidewire into the ureteral catheter. Slide and rotate the hydrophilic guidewire to detect the ureteral lumen. Once the correct ureteral lumen is found, single-J stent can be successfully placed with the guidance of hydrophilic guidewire $(18,19)$. If all methods above do not work, percutaneous nephrostomy is suggested to perform with the guidance of ultrasonography. If the single-J stent is obstructed, replace the single-J stent with a new one. Then use sterile saline to flush the single-J stent repeatedly to wash out crystals or pyuria in renal collecting system (Figure 5). Antibiotics are appropriately used based on the severity of urinary infection.

\section{Sickness caused by double- 7 stent retention}

Fully understand patients' condition (hematuria, frequency, backache or fever) and medical history (operation history, reason and time of double-J stent replacement) (20). For mild hematuria or urinary irritation symptoms, drink more water and take medicine of $\alpha$-receptor blocker or $\mathrm{M}$-cholinergic receptor blocker, and susceptible antibiotics if urinary infection exists $(21,22)$. Consider to remove double-J stent for persistent and severe hematuria. For backache caused by urine return to kidney through the double-J stent, urinate in time and avoid activity leading to urine reflux (23). For aggravated hydronephrosis or mild urinary infection, drinking more water, alkalizing urine and taking susceptible antibiotics. If pyonephrosis, fever, renal failure, or septic shock occur, it could be the complete obstruction of double-J stent. Percutaneous nephrostomy should be the first choice to relieve hydronephrosis. Choose to remove double-J stent if hydronephrosis or general health condition is not suitable for nephrostomy. Antibiotics are appropriately used based on the severity of infection.

\section{References}

12. Webb VJ, Booth CM. Cutting the cost of catheterization for acute retention--a hospital or domiciliary procedure? Br J Urol 1995;76:443-5

13. Ghaffary C, Yohannes A, Villanueva C, et al. A practical approach to difficult urinary catheterizations. Curr Urol Rep 2013;14:565-79.

14. Sliwinski A, D'Arcy FT, Sultana R, et al. Acute urinary retention and the difficult catheterization: current emergency management. Eur J Emerg Med 2016;23:80-8.

15. Paul EM, Marcovich R, Lee BR, et al. Choosing the ideal nephrostomy tube. BJU Int 2003;92:672-7.

16. Pedersen JF. Percutaneous nephrostomy guided by ultrasound. J Urol 1974;112:157-9.

17. Albrecht K, Oelke M, Schultheiss D, et al. The relevance of urinary bladder filling in suprapubic bladder catheterization. Urologe A 2004;43:178-84.

18. Kawahara T, Ito H, Terao H, et al. Ureteral stent encrustation, incrustation, and coloring: morbidity related to indwelling times. J Endourol 2012;26:178-82.

19. Krajewski W, Piszczek R, Krajewska M, et al. Urinary diversion metabolic complications - underestimated problem. Adv Clin Exp Med 2014;23:633-8.

20. Lange D, Bidnur S, Hoag N, et al. Ureteral stentassociated complications--where we are and where we are 
going. Nat Rev Urol 2015;12:17-25.

21. Wang J, Zhang X, Zhang T, et al. The role of solifenacin, as monotherapy or combination with tamsulosin in ureteral stent-related symptoms: a systematic review and meta-analysis. World J Urol 2017;35:1669-80.

22. Paick SH, Park HK, Oh SJ, et al. Characteristics of bacterial colonization and urinary tract infection after indwelling of double-J ureteral stent. Urology 2003;62:214-7.

23. Forbes C, Scotland KB, Lange D, et al. Innovations in Ureteral Stent Technology. Urol Clin North Am 2019;46:245-55. 